\title{
Se-methylselenocysteine offers selective protection against toxicity and potentiates the antitumour activity of anticancer drugs in preclinical animal models
}

\author{
S Cao ${ }^{1}$, F A Durrani ${ }^{2}, \mathrm{~K}$ Tóth $^{2}$ and Y M Rustum ${ }^{\star}, 2$ \\ ${ }^{1}$ Department of Medicine, Roswell Park Cancer Institute, Elm and Carlton Streets, Buffalo, NY 14263, USA and ${ }^{2}$ Department of \\ Cancer Biology, Roswell Park Cancer Institute, Elm and Carlton Streets, Buffalo, NY 14263, USA
}

Background: Identification and development of drugs that can effectively modulate the therapeutic efficacy and toxicity of chemotherapy remain an unmet challenge. We evaluated the effects of Se-methylselenocysteine (MSC) on the toxicity and antitumour activity of cyclophosphamide, cisplatin, oxaliplatin, and irinotecan in animal models.

Methods: Cyclophosphamide, cisplatin, and oxaliplatin were administered by a single i.v. injection and irinotecan by i.v. weekly $\times 4$ schedules. For the combination, MSC was administered daily via the oral route for 7 days in mice and daily for 14 days in rats before and concurrent with drug administration.

Results: Se-methylselenocysteine significantly protected against organ-specific toxicity induced by lethal doses of cyclophosphamide, cisplatin, oxaliplatin, and irinotecan. These include diarrhoea, stomatitis, alopecia, bladder, kidney, and bone marrow toxicities. Protection from lethal toxicity by MSC was associated with enhanced antitumour activity in rats bearing advanced Ward colorectal carcinoma and in nude mice bearing human squamous cell carcinoma of the head and neck, FaDu, and A253 xenografts.

Conclusions: Se-methylselenocysteine offers selective protection against organ-specific toxicity induced by clinically active agents and enhances further antitumour activity, resulting in improved therapeutic index. These data provided the rationale for the need to clinically evaluate MSC as selective modulator of the antitumour activity and selectivity of anticancer drugs.

Chemotherapy-induced toxicity is often a deterrent to cancer therapy, limiting treatment duration and dose escalation. Cyclophosphamide (CTX) is an alkylating agent used for treatment of haematological and solid tumours. It is a noncytotoxic prodrug with a complex metabolism that requires microsomal liver enzymes, cytochrome P450 (CYP), for conversion to its active metabolite, 4-hydroxy-cyclophosphamide (Chen et al, 2004). Efficacy is limited by its haemorrhagic cystitis (Fraiser et al, 1991; Miller et al, 1994). Severe haemorrhagic cystitis can result in bladder perforation, peritonitis, and death. Currently, sodium2-mercaptoethanesulphonate (MESNA) and hyperhydration are being used to protect against CTX-induced haemorrhagic cystitis with limited success (Shepherd et al, 1991). Alopecia is another dose-limiting toxicity induced by CTX.

Cisplatin (cis-diamminedichloroplatinum (CDDP)) is a heavy metal complex used in the treatment of a variety of malignancies. The antitumour activity of CDDP is associated with the DNA synthesis via formation of intrastrand and interstrand DNA 
crosslinks. The known toxicities of CDDP are nephrotoxicity, myelotoxicity, neurotoxicity, ototoxicity, and gastrointestinal toxicity. Nephrotoxicity is the major dose-limiting toxicity. The primary route of excretion of CDDP is through the kidneys, and it accumulates in the renal cortex, resulting in renal insufficiency, hypokalaemia, and hypomagnesaemia.

Abnormal conditions like inadequate renal function, uncompensated cardiac conditions, or generalised oedema may contraindicate the hydration regimen used to mitigate bladder and kidney toxicities of CTX and CDDP, requiring other approaches. The antioxidant compounds such as curcumin and selenium have been known to have a protective effect against kidney toxicity induced by CDDP (Baldew et al, 1989; Satoh et al, 1989; Hu et al, 1997; Antunes et al, 2000, 2001; Chen et al, 2004). Amifostine was approved by the US Food and Drug administration for reducing the cumulative renal toxicity associated with repeated CDDP treatment.

Oxaliplatin (ELOXATIN) is an active anticancer drug approved by the US FDA for the treatment of advanced colorectal cancer (Ibrahim et al, 2004). Oxaliplatin is an alkylating agent that forms intrastrand links between two adjacent quinine residues or a guanine and adenine, hence disrupting DNA replication and transcription (Bleiberg, 1998). It is the only platinum compound active against colorectal cancer (Cao et al, 2006). Oxaliplatin has been mainly used in combination with other chemotherapeutic drugs, including irinotecan (Wasserman et al, 2001) and 5-FU/LV (Alberts et al, 2005), with significantly higher activity. The doselimiting toxicity of oxaliplatin in the clinic includes neuropathy, myelotoxycity diarrhoea, and mucositis (Wilson et al, 2002; Cao et al, 2006). Several approaches that have been utilised clinically to manage toxicities include dose reduction, schedule alternation, and delay of therapy. As it was not feasible to quantitate neurotoxicity in rats treated with oxaliplatin, and as diarrhoea and stomatitis are the common clinical toxicities, the effects of Se-methylselenocysteine (MSC) on these toxicities were assessed in the rat model.

Irinotecan (CPT-11) is a topoisomerase I poison active in a variety of solid tumour malignancies, used in combination for the treatment of patients with advanced colorectal cancer. Irinotecan is enzymatically converted by caboxylesterase (CES) 1 and 2 to 7-Ethyl-10 hydroxy camptothecin (SN-38), the therapeutically active metabolite. Conversion by UDP-glucuronosyl transferase 1A1 (UGT1A1) results in SN-38 glucuronide (SN-38G) that gets excreted in bile and intestines. Delayed-onset diarrhoea is a dose-limiting toxicity for irinotecan (Bleiberg, 1998). Despite use of high-dose loperamide to prevent CPT-11-induced diarrhoea, the efficacy is limited (Bleiberg, 1998). Neutropenia is another major adverse event for irinotecan treatment with transient neutropenia occurred in $80 \%$ of patients, and severe neutropenia and febrile neutropenia in $47 \%$ and $15 \%$, respectively (Bleiberg, 1998). As the data in Figures 5 and 6 demonstrates, the antitumour activity of irinotecan is host, selenium and tumour-type dependent, and this drug is used clinically in head and neck cancer with myelosuppression and diarrhoea as the dose-limiting toxicities. The effects of MSC on irinotecan toxicity profile and antitumour activity were assessed in this study.

Selenium, an essential trace element and antioxidant, is known to be used in the prevention of various cancers (Clark et al, 1991, 1996; Zhuo et al, 2004; Brinkman et al, 2006; Amaral et al, 2010). Specific types of selenium, such as MSC, potentiated the therapeutic efficacy of a variety of chemotherapeutic agents against various human tumour xenografts (Cao et al, 2004). The present study was undertaken to evaluate further the effects of MSC on the toxicity and antitumour activity of several chemotherapeutic agents with documented clinical efficacy and dose-limiting toxicities. An agent, such as MSC, that can protect normal tissues from druginduced toxicities and enhance the antitumour activity offers the possibility to alter the therapeutic index of anticancer drugs. Protection against toxicities induced by chemotherapy offer the potential for the use of higher doses of chemotherapy with the hope of overcoming drug resistance.

\section{MATERIALS AND METHODS}

Animals. Eight to 12-week-old female Fischer 344/N rats (body weight $150-180 \mathrm{~g}$ ) and female athymic nude mice (nu/nu, body weight 20-25 g) were obtained from Harlan Sprague Dawley Inc. (Indianapolis, IN, USA) and kept four rats or five mice per cage with water and food ad libitum according to an institutionally approved animal protocol. There were 4 rats per group for a single experiment in a total of 12-24 rats in each group from 3 to 6 independent experiments, whereas 5 mice were used in each experimental group in a total of 10 mice in each group from 2 independent experiments. All studies were performed in accordance with the protocols approved by the Institutional Animal Care and Use Committee.

Tumours. (1) Ward colorectal carcinoma: the chemically induced tumour in Fischer rats was extensively used in this laboratory (Cao and Rustum, 2000). Nonnecrotic tumour pieces ( 100 mg) were transplanted subcutaneously (s.c.) to the flanks of rats via trocar under slight anaesthesia. Treatment was initiated 14 days later when the tumours reached $\sim 2500-3000 \mathrm{mg}$. (2) FaDu and A253 human head and neck squamous cell carcinomas (HNSCCs): the HNSCC xenografts were initially established in nude mice from cultured cells by inoculating s.c. $10^{6}$ cells in the flank of the mice. The xenografts were subsequently passed several generations by transplantation of $\sim 50 \mathrm{mg}$ nonnecrotic tumour tissues in the flank of mice before treatment (Cao et al, 1999). Treatment was initiated 7 days later when the tumours reached 200-220 mg.

Drugs. Se-methylselenocysteine was purchased from Sigma (St Louis, MO, USA) and dissolved in sterile saline $(0.9 \% \mathrm{NaCl})$ at a concentration of $1 \mathrm{mg} \mathrm{ml}^{-1}$. Cyclophosphamide and CDDP (Bristol-Myers Squibb Co., Princeton, NJ, USA) were used as a ready-to-use clinical formulation solution in $50-100 \mathrm{ml}$ vials that contained $1000 \mathrm{mg}$ of CTX $(20 \mathrm{mg} \mathrm{ml})$ and $100 \mathrm{mg}$ of CDDP $\left(1 \mathrm{mg} \mathrm{ml}^{-1}\right)$, respectively. Oxaliplatin (ELOXATIN) was purchased from Sanofi-Synthelabo (New York, NY, USA) as a solution of $5 \mathrm{mg} \mathrm{ml}^{-1}$ in $10 \mathrm{ml}$ vials. Irinotecan was purchased from Pfizer Inc. (New York, NY, USA) as a ready-to-use clinical formulation solution in $5 \mathrm{ml}$ vials containing $100 \mathrm{mg}$ of the drug $\left(20 \mathrm{mg} \mathrm{ml}^{-1}\right)$.

Drug doses and schedules. Se-methylselenocysteine was administered by the oral route (p.o.) daily at various doses (from 0.25 to $1.25 \mathrm{mg}$ per rat per day) daily for up to 35 days for the rats and at a fixed dose ( $0.2 \mathrm{mg}$ per mouse per day) daily for 14 days for the mice. Cyclophosphamide (from 50 to $200 \mathrm{mg} \mathrm{kg}^{-1}$ ), CDDP (from 3 to $12 \mathrm{mg} \mathrm{kg}^{-1}$ ), and oxaliplatin (from 5 to $25 \mathrm{mg} \mathrm{kg}^{-1}$ ) were administered by a single i.v. injection via the animals' tail vein, whereas irinotecan (100-200 $\mathrm{mg} \mathrm{kg}^{-1}$ per week) was administered by i.v. weekly $\times 4$ (once a week for 4 weeks). For the combination of MSC and CTX, CDDP, or oxaliplatin, MSC was orally administered daily for 14 days (for rats) or 7 days (for mice) before chemotherapy and for 7 days thereafter. For the combination of MSC and irinotecan, MSC was given orally for 35 days in total. The schedule used for MSC was based on our early studies (Cao et al, 2004) in mice, wherein the optimal effect of antitumour efficacy and toxicity protection occurred only when MSC was given at least 7 days before and concurrent with administration of chemotherapeutic agent.

Maximum tolerated dose (MTD) and toxicity assessment. The MTD was defined as the maximum dose administered to the 
animals that results in no drug-related lethality and body weight loss of $<20 \%$. Animals were monitored daily for drug-induced toxicity (body weight loss, diarrhoea, stomatitis, and lethality) for a minimum of 2 weeks, and 3-4 times a week thereafter. Each treatment group had 12-24 rats (3-6 experiments) and at least 10 mice ( 2 experiments) in total.

Alopecia evaluation. Studies were carried out by observing hair regrowth after shaving of rats. Briefly, areas on the back of rats (measuring $\sim 7 \times 9 \mathrm{~cm}$ ) were shaved and the animals were randomised into different groups of untreated control, CTX alone, and CTX plus MSC. Cyclophosphamide was administered at the MTD $\left(100 \mathrm{mg} \mathrm{kg}^{-1}\right.$, i.v. $\left.\times 1\right)$ and MSC was given $0.75 \mathrm{mg}$ per rat per day p.o. daily for 21 days, with the first dose having started 14 days before CTX treatment. Animals were evaluated on days 0,30 , and 45 after CTX and followed up for 90 days. Complete hair regrowth after shaving occurred within 30-45 days in control animals. Four rats were used in each experimental group and treatments were repeated three times.

Genitourinary (GU) toxicity evaluation. As the acute toxicity of GU occurs within few hours of drug administration, rat bladders were removed at $4 \mathrm{~h}$ and $24 \mathrm{~h}$ post CTX. The toxicity effects of CDDP on the kidneys become evident at a later time point; hence, kidneys were removed on day 6 after CDDP administration. Blood samples were also collected post mortem by cardiac puncture from the same rats. Sera obtained from the blood samples were used for blood urea nitrogen (BUN) and serum creatinine determination using commercial kits (Ortho-Clinical Diagnostics, Rochester, NY, USA) according to the manufacturer's instructions. Kidney and bladder specimens (nine rats for each group) were fixed in $10 \%$ neutral buffered formalin, embedded in paraffin, and $5 \mu \mathrm{m}$ sections were stained with conventional haematoxylin-eosin for histopathological study. The BUN levels were measured using urease method with commercially available Vitros BUN/UREA slides on an Ortho-Clinical Diagnostics - Vitros 500 chemistry analyzer (Ortho-Clinical Diagnostics). Creatinine was assayed using the creatinineamidohydrolase method and Vitros CREA slides (OrthoClinical Diagnostics) on an Ortho-Clinical Diagnostics - Vitros 500 chemistry analyzer (Spayd et al, 1978). The examined groups included untreated control, MSC (0.75 mg per rat per day), CTX $\left(100\right.$ or $150 \mathrm{mg} \mathrm{kg}^{-1}$, i.v. $\left.\times 1\right)$ or CDDP $\left(6 \mathrm{mg} \mathrm{kg}^{-1}\right.$, i.v. $\left.\times 1\right)$ alone, and CTX $\left(100\right.$ or $150 \mathrm{mg} \mathrm{kg}^{-1}$, i.v. $\left.\times 1\right)$ or CDDP $\left(6 \mathrm{mg} \mathrm{kg}^{-1}\right.$, i.v. $\left.\times 1\right)$ plus $\mathrm{MSC}(0.75 \mathrm{mg}$ per rat per day $)$. For the combination group of CTX or CDDP and MSC, MSC was given 14 days before CTX or CDDP treatment and continuous daily until the rats were killed Nine rats were evaluated in each group from two independent experiments (three rats for the first experiment and six rats for the second experiment).

Tumour measurement and antitumour activity evaluation. The animals were randomly divided into different groups of 4 rats or 5 mice each and initiated treatment with CTX or CDDP when the tumours reached $\sim 2500-3000 \mathrm{mg}\left(\mathrm{mm}^{3}\right.$ ) in the rats (14 days after tumour transplantation) and $200-250 \mathrm{mg}$ in the mice (7 days after tumour transplantation), whereas MSC was initiated 14 days for the rats and 7 days for the mice before CTX, CDDP, oxaliplatin, or irinotecan treatment at the same day of tumour transplantation and 7 days after treatment. Two axes of the tumour (L, longest axis; $\mathrm{W}$, shortest axis) were measured with a Vernier caliper. Tumour weight $(\mathrm{mg})$ or volume $\left(\mathrm{mm}^{3}\right)$ was calculated as: $1 / 2\left(\mathrm{~L} \times \mathrm{W}^{2}\right)(\mathrm{mm})$. Measurements were taken once a day during the treatment and 2 to 3 times a week thereafter. Tumour response was expressed as a partial response (PR) when tumour weight was temporarily reduced by at least $50 \%$ compared with initial tumour size, and as a complete response (CR) when tumour was undetectable by palpation at the site of transplant. Cure was defined as animal surviving with no tumour at the end of the 60-day period, at which time the animals were humanely killed.

Histopathological studies. Routine haematoxylin and eosin staining was used on formalin-fixed, paraffin-embedded tissues. Histopathological changes of tissues after the treatment of CTX or CDDP alone or in combination with MSC were analysed (by Károly Tóth, a board-certified pathologist) and compared with untreated controls (nine rats for each group) to determine druginduced toxicity and protective effect of MSC.

Statistical analysis. The differences between the mean values in the different treatment groups were analysed for significance using one-way analysis of variances (ANOVA) with Tukey-Kramer multiple comparison tests. A $P$-value of $<0.05$ was considered to be statistically significant.

\section{RESULTS}

MSC protects against toxicities induced by chemotherapeutic agents in Fischer rats. Although the MTD of MSC with tested schedules in normal and tumour-bearing rats was $0.75 \mathrm{mg}$ per rat per day (administered for up to 42 days), a single dose of $1.5 \mathrm{mg}$ per rat was highly toxic. Thus, the dose of $0.75 \mathrm{mg}$ per rat per dose of MSC was used for evaluating the protective effects on toxicities induced by various chemotherapeutic agents. The data demonstrated that except for CTX, MSC offered complete protection from the toxicities induced by lethal doses of CDDP, oxaliplatin, and irinotecan, results consistent with our previous findings with nude mice (Cao et al, 2004). The optimal protection from chemotherapy-induced toxicity was achieved only when MSC treatment preceded drug treatment. The protective effects of MSC on diarrhoea, stomatitis (mouth ulceration), and lethality induced by chemotherapy are summarised in Table 1.

The incidences of sustained diarrhoea and stomatitis were used as indicators of GI toxicity induced by chemotherapeutic agents. Although no significant toxicities were observed at the MTD of CTX (100 $\mathrm{mg} \mathrm{kg}^{-1}$ ), 50\%, 38\%, and 62\% of animals developed severe diarrhoea, stomatitis, and lethality at the toxic dose of $150 \mathrm{mg} \mathrm{kg}^{-1}$. Se-methylselenocysteine completely prevented the development of both diarrhoea and stomatitis (0\%) and reduced the lethality from $62 \%$ to $25 \%$ induced by CTX. At the MTD of CDDP $\left(6 \mathrm{mg} \mathrm{kg}^{-1}\right), 50 \%$ of animals developed diarrhoea with no lethality. In contrast, MSC completely protected animals from diarrhoea at $6 \mathrm{mg} \mathrm{kg}^{-1}$ of CDDP. Similarly, although rats treated with CDDP at $9 \mathrm{mg} \mathrm{kg}^{-1}$ induced severe diarrhoea (100\%) and stomatitis (75\%) and resulted in 100\% lethality, MSC pretreatment resulted in complete protection against diarrhoea, stomatitis, and lethality. Similar protection was achieved from the toxicities induced by oxaliplatin and irinotecan.

MSC protects against CTX-induced bladder toxicity. Lethality was evident within 6-8 days with toxic doses of CTX, whereas rats that recovered from the acute toxicity did not show any evidence of delayed toxicity. As no significant toxicity was observed with the MTD of CTX (100 $\mathrm{mg} \mathrm{kg}^{-1}$ ) alone and in combination with MSC, the dose of CTX of $150 \mathrm{mg} \mathrm{kg}^{-1}$ was used to document the protective effect of MSC. Four groups (nine rats for each group) consisting of untreated control, MSC alone, CTX alone, and MSC plus CTX were evaluated. Acute bladder toxicity was evident histopathologically with the CTX alone. The representative morphologic pictures of bladder are illustrated in Figure 1. Figure $1 \mathrm{~A}$ shows the characteristic preserved normal histological features of a rat bladder in control (untreated group), with no significant histological change observed when MSC was used alone (Figure 1B). Figure 1C shows the presence of acute mucosal oedema in the bladder mucosa at $4 \mathrm{~h}$ post CTX administration that 


\begin{tabular}{|c|c|c|c|c|}
\hline \multirow[b]{2}{*}{ Treatment (mg kg ${ }^{-1}$ ) } & \multicolumn{4}{|c|}{ Toxicity (\% of total) } \\
\hline & $M W L^{a}$ & Diarrhoea & Stomatitis & Lethality \\
\hline Control & $2.1 \pm 0.6$ & 0 & 0 & 0 \\
\hline MSC (0.75) & $5.8 \pm 1.2$ & 0 & 0 & 0 \\
\hline CTX (100) & $9.9 \pm 1.6$ & 0 & 0 & 0 \\
\hline CTX (100) + MSC (0.75) & $6.6 \pm 3.0^{*}$ & 0 & 0 & 0 \\
\hline CTX (150) & $18.5 \pm 3.0$ & 50 & 38 & 62 \\
\hline CTX (150) + MSC (0.75) & $16.6 \pm 1.2^{*}$ & 0 0** & 0 0** & $25^{\star \star}$ \\
\hline $\operatorname{CDDP}(6)$ & $13.5 \pm 2.4$ & 50 & 0 & 0 \\
\hline $\operatorname{CDDP}(6)+\mathrm{MSC}(0.75)$ & $10.6 \pm 2.1^{*}$ & 0 0*** & 0 & 0 \\
\hline $\operatorname{CDDP}(9)$ & $21.9 \pm 2.8$ & 100 & 75 & 100 \\
\hline $\operatorname{CDDP}(9)+\mathrm{MSC}(0.75)$ & $13.5 \pm 2.4^{\star \star}$ & $0 * \star \star$ & $0^{\star \star \star}$ & $0 \star 0 \star \star$ \\
\hline Oxal (20) & $20.8 \pm 5.3$ & 0 & 0 & 100 \\
\hline Oxal (20) + MSC (0.75) & $9.4 \pm 2.6$ & 0 & 0 & 0 \\
\hline Irinotecan (200) & $22.5 \pm 3.2$ & 30 & 0 & 100 \\
\hline Irinotecan (200) + MSC (0.75) & $10.5 \pm 1.5$ & 0 & 0 & 0 \\
\hline \multicolumn{5}{|c|}{ 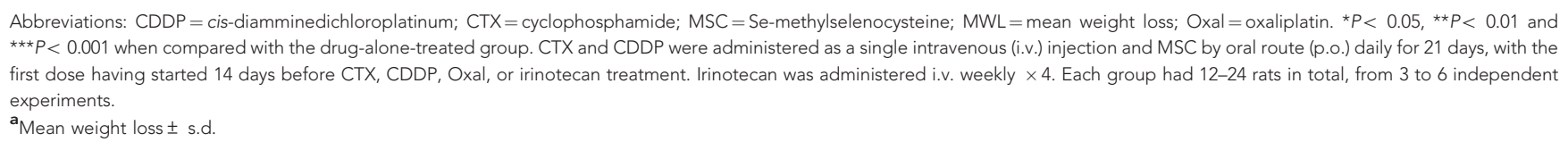 } \\
\hline
\end{tabular}

progressed by $24 \mathrm{~h}$ to severe haemorrhagic cystitis (arrows, Figure 1D), involving destruction of the bladder mucosa. Se-methylselenocysteine significantly protected from bladder toxicity induced by CTX with only a minor oedema and with the epithelium preserved at $2 \mathrm{~h}$ post CTX administration (Figure 1E) and prevented the progress to haemorrhagic cystitis at $24 \mathrm{~h}$ (Figure $1 \mathrm{~F}$ ) as seen in CTX alone (Figure 1D). The complete lack of haemorrhage in the group of MSC plus CTX documents the protective effects of MSC from CTX-induced bladder toxicity.

MSC protects rats from CDDP-induced renal toxicity. Nephrotoxicity is the dose-limiting toxicity of CDDP in the clinic. The data in Figure 2 (upper) summarise the effect of MSC on the renal function as measured by blood BUN and creatinine. Treatment of CDDP at the MTD $\left(6 \mathrm{mg} \mathrm{kg}^{-1}\right.$, i.v. $\left.\times 1\right)$ caused a significant increase in BUN and creatinine levels compared with controls $(P<0.001)$. In contrast, the combination of MSC and CDDP prevented the increases with similar levels of BUN and creatinine compared with the control group $(P>0.05)$. These data demonstrate that MSC can effectively protect against CDDP-induced kidney toxicity. Histopathological examination of the kidneys of rats (nine rats for each group) from the groups of untreated control, MSC alone, CDDP alone, and CDDP in combination with MSC was carried out and the representative results are shown in Figure 2 (bottom). Figure $2 \mathrm{~A}$ and $\mathrm{B}$ shows the normal morphology of kidneys of rats from untreated controls and treated with MSC, respectively. Figure $2 \mathrm{C}$ and $\mathrm{D}$ shows the kidneys of rats treated with CDDP alone and in combination with MSC respectively. The picture in Figure 2C shows dilated tubules because of destruction of the kidney's tubular epithelium by CDDP, whereas the data in Figure 2D show near-normal kidney histology because of the protective effect of MSC. In the group of 9 rats treated with the combination of CDDP and MSC, 8 animals (89\%) showed $3-5 \%$ of total tubules that were slightly dilated and lined with flat, instead of cubic, epithelium. Only 1 of 9 animals (11\%) showed near-normal histological features in the group treated with CDDP alone $(P<0.001)$.

MSC protects rats from CTX-induced alopecia. Figure 3 depicts the effect of CTX alone and in combination with MSC on alopecia in rats on days 0 (upper panels), 30 (middle panels), and 45 (bottom panels). Cyclophosphamide was given at the MTD $\left(100 \mathrm{mg} \mathrm{kg}^{-1}\right.$, i.v. $\times 1)$, and MSC $(0.75 \mathrm{mg}$ per rat per day, p.o.) was administered daily for 21 days, with the first dose having started 14 days before CTX (100 $\left.\mathrm{mg} \mathrm{kg}^{-1}\right)$ for the combination. Treatment with MSC alone did not induce alopecia in this model system. The first column demonstrates the recovery of body hair in untreated control over 30-45 days after shaving. Column 2 shows a slow and partial recovery of body hair after shaving in rats treated with CTX alone, whereas column 3 shows the body hair recovery comparable to the untreated controls in rats treated with the combination of MSC and CTX. The result demonstrates the effectiveness of MSC in protecting and promoting hair growth after CTX treatment. Twelve rats from three different experiments were evaluated for each group and similar results were observed, although only two representative pictures are shown for each group.

MSC is highly effective in the protection of bone marrow toxicity induced by oxaliplatin. Figure 4 (upper) shows that a single dose of oxaliplatin $\left(20 \mathrm{mg} \mathrm{kg}^{-1}\right.$ i.v.) higher than the MTD induced very severe myelotoxicity, to basically 'wipe out' the bone marrow ( $\sim 98 \%$ myelosuppression) on day 8 after the treatment. The same treatment combined with MSC resulted in the modest protection with $\sim 80 \%$ myelosuppression. Administration of a single dose of oxaliplatin at the MTD $\left(15 \mathrm{mg} \mathrm{kg}^{-1}\right)$ induced $\sim 90 \%$ and $\sim 20-30 \%$ myelosuppression estimated in the bone marrow from sternum on days 8 and 24 after administration, respectively. When the same dose of oxaliplatin $\left(15 \mathrm{mg} \mathrm{kg}^{-1}\right)$ was combined with MSC, the myelosuppression in bone marrow from sternum decreased to $\sim 40-60 \%$ on day 8 after oxaliplatin administration 


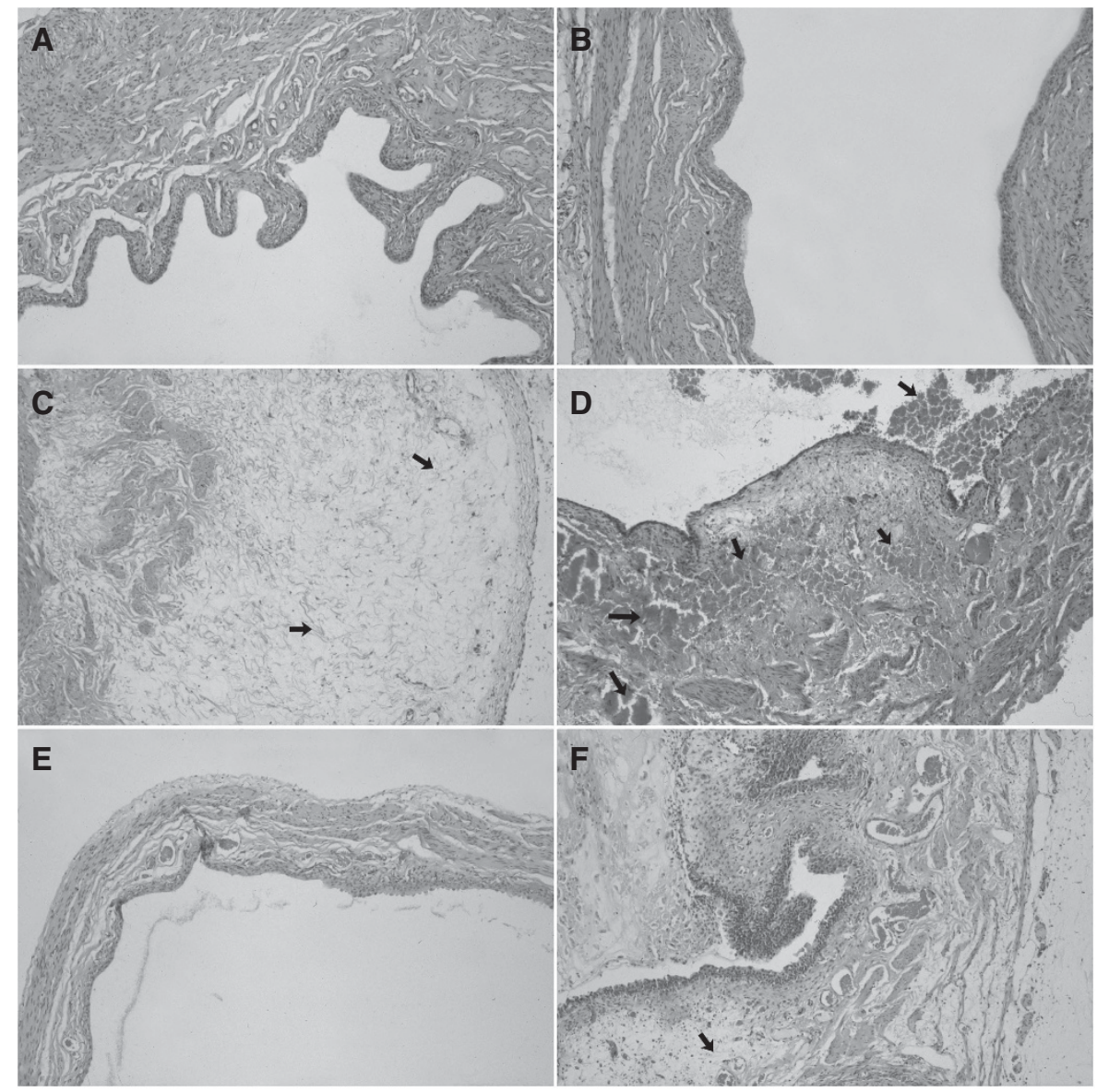

Figure 1. Protective effects of MSC on CTX-induced rat bladder toxicity. Photomicrographs show rat bladder with untreated control, MSC alone, CTX alone, or CTX plus MSC. Cyclophosphamide was administered as a single i.v. injection at $150 \mathrm{mg} \mathrm{kg}^{-1}$ (toxic dose) and MSC at $0.75 \mathrm{mg}$ per rat per day by p.o. daily for 14 days before CTX treatment. The bladders were removed at 4 and $24 \mathrm{~h}$ after CTX treatment. Conventional formalinfixed paraffin-embedded sections were stained with haematoxylin and eosin. In order to see large parts of the bladder mucosa, (A, B, and E) are $\times 100$; in order to see more histological details, $($ C, D, and F) are $\times 200$. (A) Histologic picture of an untreated normal bladder with preserved histological features. (B) No change with MSC alone. Photomicrograph of rat bladder treated with CTX shows an acute mucosal oedema (arrow) at $2 \mathrm{~h}$ (C) that progressed to characteristic, severe haemorrhagic cystitis (arrows) at $24 \mathrm{~h}$ (D). Se-methylselenocysteine protected from CTX-induced acute mucosal oedema as seen on a long bladder segment with preserved normal histological structure (E). The haemorrhages in the mucosa and in the bladder wall were completely prevented by MSC (F). Oedema (arrow) is still present, but is considered a minor lesion as compared with haemorrhages seen with CTX alone without MSC. Each group had nine rats from two independent experiments.

and, moreover, in $\sim 2$ more weeks, on day 24 after oxaliplatin administration, the bone marrow from sternum was completely restored.

Figure 4 (bottom) visualises the oxaliplatin-induced myelotoxicity and its prevention with MSC in representative microphotographs of the histological slides of bone marrow from rat sternum. Figure 4 panel 1 shows the normal rat bone marrow that always contains $\sim 20-30 \%$ fat tissue. Panel 2 of Figure 4 demonstrates that after MSC treatment the myelopoiesis was intact, and there was no sign on myelotoxicity. Figure 4 panel 3 shows that after the single dose of oxaliplatin at the MTD $\left(15 \mathrm{mg} \mathrm{kg}^{-1}\right)$ on day 24 after the administration, the haematopoietic area decreased significantly and was replaced by a much larger extension of the fat tissue as compared with the control. Figure 4 panel 4 visualises that the single dose of oxaliplatin at the MTD $\left(15 \mathrm{mg} \mathrm{kg}^{-1}\right)$ combined with MSC restored the normal haematopoiesis and prevented the oxaliplatin-induced myelotoxicity on day 24 after administration.

MSC potentiates the antitumour activity of CDDP and oxaliplatin in rats bearing advanced colorectal carcinoma and/or in nude mice bearing human FaDu and A253 head and neck xenografts. After we demonstrated significant protection by
MSC of toxicities induced by CTX and CDDP in rats, studies were carried out to determine the selective effects of MSC in rats bearing advanced ( 2500-3000 mg) Ward colorectal carcinoma and in nude mice bearing human FaDu and A253 head and neck cancer xenografts.

The data in Figure 5A show the effects of MSC on the antitumour efficacy of CDDP, oxaliplatin, and irinotecan in rats bearing advanced Ward colorectal carcinoma. The data demonstrated that rats treated with MSC produced 30\% tumour growth inhibition with no complete regression (CR), and pretreatment with MSC enhanced significantly the CR of rats bearing advanced tumours. For example, CDDP, at its MTD $\left(6 \mathrm{mg} \mathrm{kg}^{-1}\right)$ yielded $32 \%$ CR (cure) and this CR rate was increased to $75 \%$ when it was used in combination with MSC. The data in Figure 5A also demonstrated that the administration of MSC in combination with the lethal dose of CDDP $\left(9 \mathrm{mg} \mathrm{kg}^{-1}\right)$ resulted in $75 \% \mathrm{CR}$ rate with no toxicity to normal tissues. These data support the previously documented hypothesis obtained in other tumour models (Cao et al, 2004) that MSC protection of normal tissues allowed the administration of higher than the MTD doses of CDDP and oxaliplatin, resulting in higher response rate. Although the 50\% CR rate achieved with oxaliplatin was not dose dependent (Figure $5 \mathrm{~A}$, solid bar), the combination with MSC resulted in $100 \% \mathrm{CR}$. This 


\begin{tabular}{lcc}
\hline Treatment & $\mathrm{BUN}\left(\mathrm{mg} \mathrm{dl}^{-1}\right)$ & Creatinine $\left(\mathrm{mg} \mathrm{dl}^{-1}\right)$ \\
\hline Control & $14.7 \pm 2.1$ & $0.34 \pm 0.05$ \\
MSC $(0.75 \mathrm{mg} \mathrm{per}$ day $)$ & $12.7 \pm 1.2$ & $0.30 \pm 0.00$ \\
CDDP $\left(6 \mathrm{mg} \mathrm{kg}^{-1}\right)$ & $197.0 \pm 112.8$ & $4.62 \pm 3.50$ \\
CDDP $\left(6 \mathrm{mg} \mathrm{kg}^{-1}\right)+$ MSC $(0.75 \mathrm{mg}$ per day $)$ & $13.7 \pm 3.9^{2}$ & $0.41 \pm 0.06^{2}$ \\
\hline
\end{tabular}

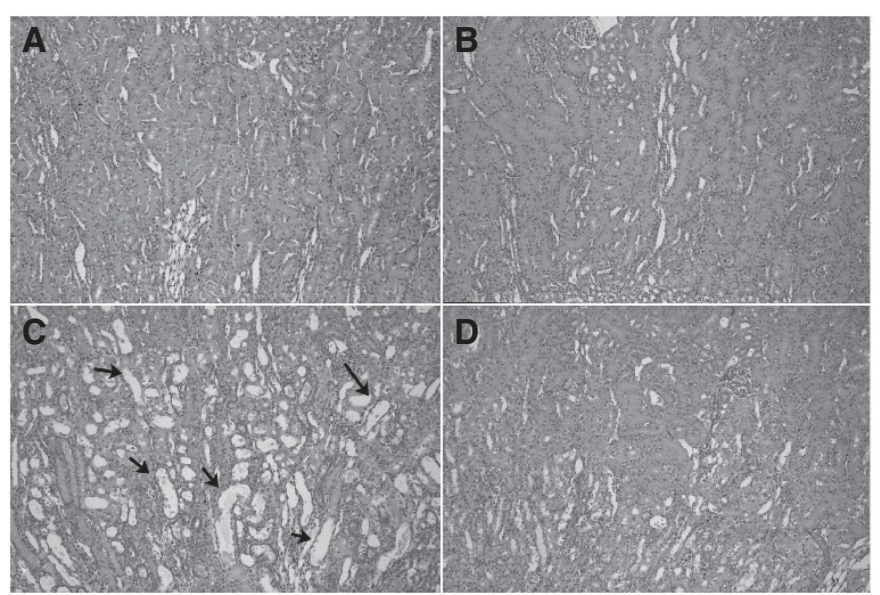

Figure 2. Histological demonstration of the protective effects of MSC against rat kidney toxicity induced by CDDP treatment in Fischer rats. Kidneys of rats were taken on day 6 from the treatment of MSC alone, CDDP alone, CDDP plus MSC, and untreated controls. The CDDP was administered as a single i.v. injection at $6 \mathrm{mg} \mathrm{kg}^{-1}$ (MTD) and MSC $0.75 \mathrm{mg}$ per rat per day by p.o. daily for 20 days, with the first dose having started 14 days before CDDP treatment. Conventional formalin-paraffin sections were stained using haematoxylin and eosin $(\times 100$

magnification). Representative photomicrograph in (A) shows the histomorphology of normal kidney from an untreated rat; (B) shows no any histomorphological change by MSC alone; (C) shows that CDDP alone induced severe tubular damage with irregular, dilated proximal, and distal tubules (arrows) lined with flat epithelium; MSC significantly protected from CDDP-induced toxicity with almost normal kidney histology in the rat treated with MSC plus CDDP in (D). Each group had nine rats in total from two independent experiments (three rats for each group from the first experiment and six rats for each group from the second experiment). Pictures are shown of only one representative kidney for each group.
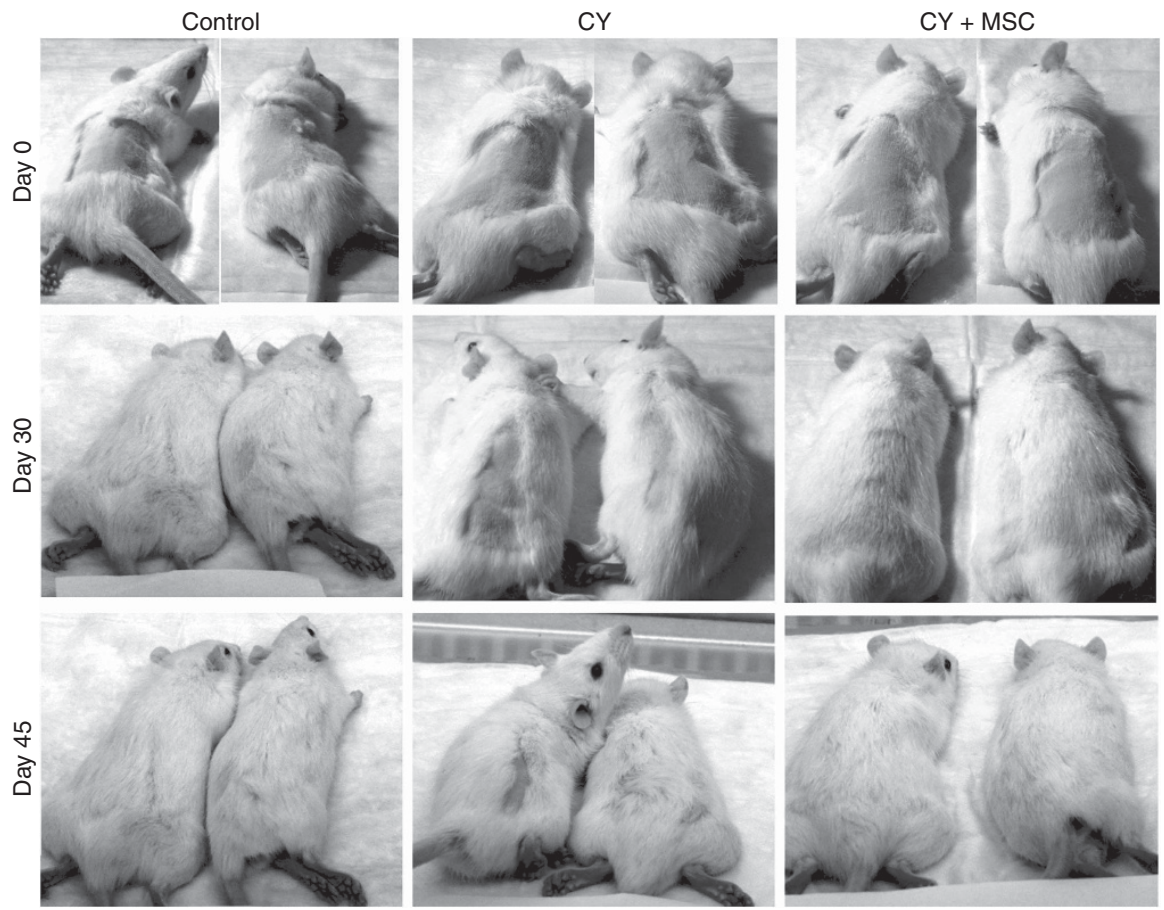

Figure 3. Se-methylselenocysteine (MSC) protects against CTX-induced alopecia in rats. Assessment was carried out on day 0 (row 1 ), day 30 (row 2), and day 45 (row 3) after CTX treatment. Column 1 of the photograph shows uneventful growth of body hair in untreated control over $30-45$ days. Column 2 shows tardy partial hair growth in rats treated with CTX alone. Column 3 shows normal hair growth in rats treated with the combination of MSC and CTX. Cyclophosphamide was administered as a single i.v. injection at $100 \mathrm{mg} \mathrm{kg}^{-1}$ (MTD) and MSC $0.75 \mathrm{mg}$ per rat per day by p.o. daily for 21 days, with the first dose having started 14 days before CTX treatment. Each group had 12 rats in total, from 3 independent experiments (pictures shown for only two representative rats for each group). 


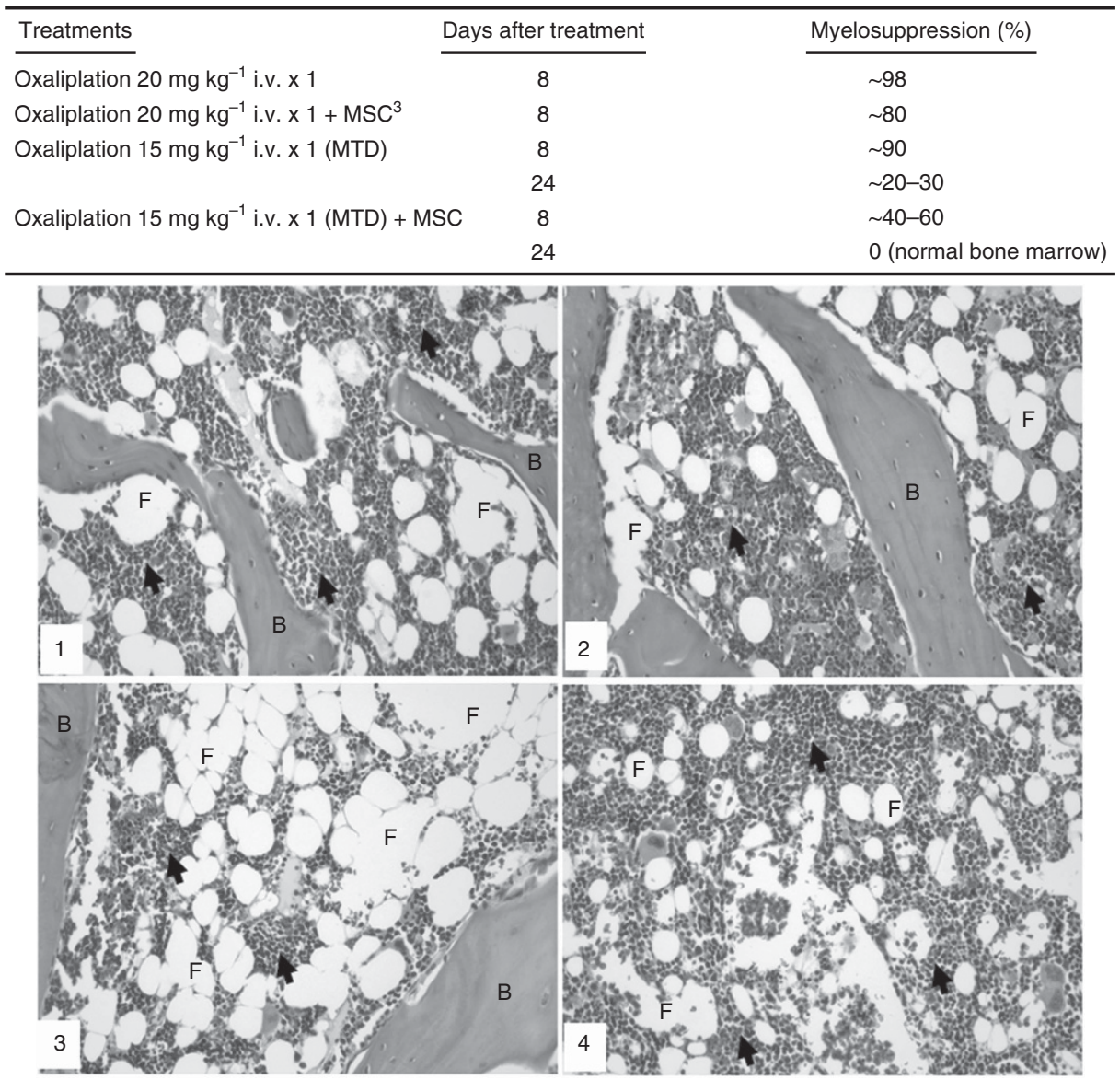

Figure 4. Se-methylselenocysteine (MSC) protects against myelotoxicity in bone marrow induced by oxaliplatin, histopathological study in Fischer rats. The rats were treated with oxaliplatin at the MTD $\left(15 \mathrm{mg} \mathrm{kg}^{-1}\right.$ i.v. $\left.\times 1\right)$ with or without MSC at $0.75 \mathrm{mg}$ per rat per day by p.o. daily for 21 days, with the first dose having started 14 days before oxaliplatin treatment. The sternums were removed on days 8 and 24 after oxaliplatin treatment and conventional formalin-paraffin sections of the bone marrow from sternum were decalcified and stained with haematoxylin and eosin. Representative photomicrographs of rat bone marrow are shown. All photographs were taken at $\times 200$ magnification. (1) histomorphology of untreated normal bone marrow between bone trabeculae (B). The bone marrow (arrows) is infiltrated with fat (F) tissue as part of the normal histology. (2) MSC alone did not cause any change in the histological structure of the bone marrow. (3) Oxaliplatin $\left(15 \mathrm{mg} \mathrm{kg}^{-1}\right.$ i.v., $\left.\times 1\right)$ alone induced severe myelosuppresion ( 90\%) on day 8 (not shown), and improved on day 24 with $\sim 40-60 \%$ myelosuppression is shown in the panel. (4) On day 24, MSC completely restored the histological structure and cellular composition of the bone marrow (arrows) induced by oxaliplatin.

rat model, however, was not responsive to irinotecan alone and in combination with MSC. In contrast, MSC potentiated the antitumour activity of irinotecan in xenografts bearing human colorectal carcinomas, HT29 and HCT8. The CR rate was increased from $\sim 30 \%$ with the drug alone to $100 \%$ with the combination with MSC (Cao et al, 2004).

The data in Figure 5B is the kinetics of the antitumour activity of CTX and CDDP alone and in combination with MSC against $\mathrm{FaDu}$ and A253 xenografts bearing in nude mice. The data indicate that although MSC had no significant antitumour activity against both tumours, CTX and CDDP produced significant tumour growth inhibition $(P<0.05)$. The antitumour activity of CTX and CDDP in combination with MSC was increased significantly $(P<0.05$ compared with CTX and CDDP alone). Collectively, these data using two chemotherapeutic agents and two tumour models in two different host indicates that MSC can potentiate the antitumour efficacy and therapeutic selectivity resulting in improved therapeutic index.

The antitumour activity of selenium in combination with chemotherapy is selenium-type dependent. The inconsistent benefit from selenium prevention trials provided the basis to evaluate the antitumour activity of various selenium types administered orally at their prospective MTD, in combination with irinotecan in xenograft models. The observed $20 \%$ to $30 \%$ tumour growth inhibition by selenium-containing compounds appear to sensitise tumour cells to subsequent treatment with cytotoxic agents, and no CR was observed. The data in Figure 6 demonstrate that in these model systems, the quality of treatment outcome is selenium-type dependent. This difference in efficacy could be related to a number of factors including metabolism, protein binding capacity, and pharmacokinetics and pharmacodynamics variation.

\section{DISCUSSION}

The effectiveness of chemotherapeutic agents is limited by toxicities, lack of therapeutic selectivity, and drug resistance. Novel approaches to develop new agents and/or combination regimens to reduce toxicity and increase selectivity are clearly needed. Selenium, an important trace element that is present in various degrees and types in the diet, has been the focus of multiple chemoprevention studies (Clark et al, 1991, 1996; Ip et al, 1999). 

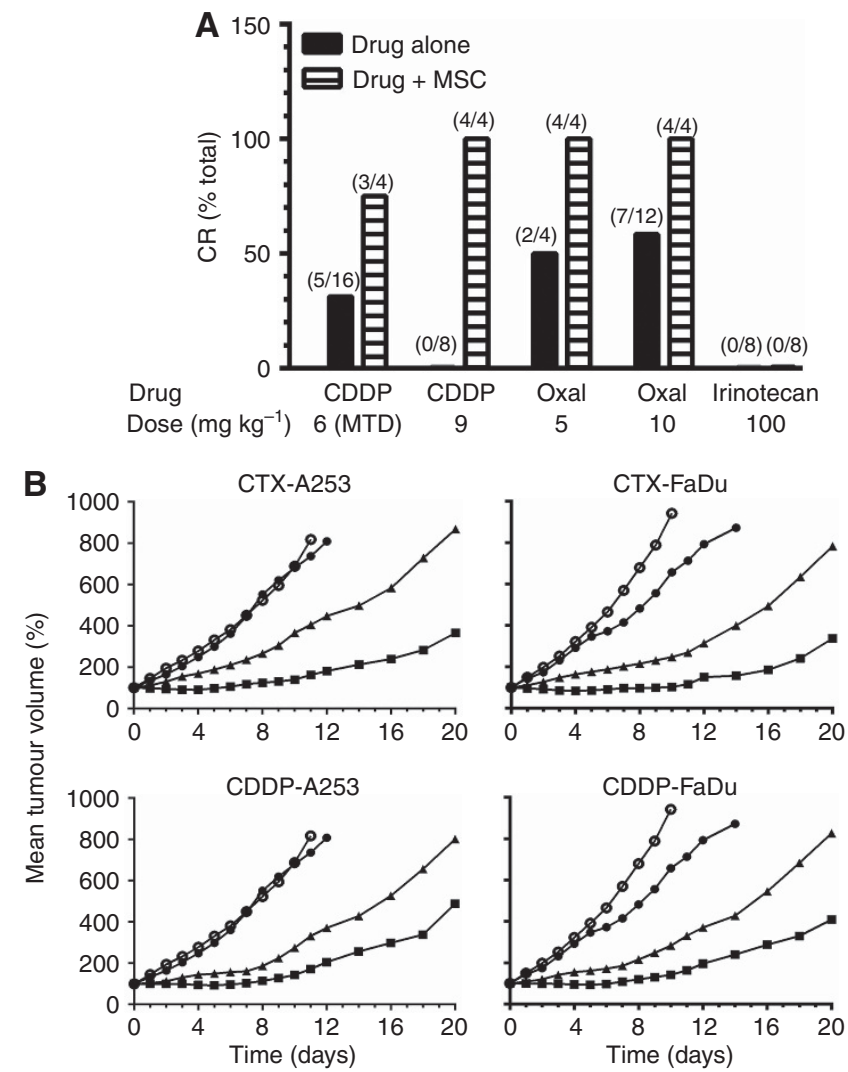

Figure 5. Antitumour activity of CDDP, oxaliplatin, and irinotecan alone and in combination with MSC in rats bearing advanced Ward colorectal carcinoma (A) and CTX and CDDP alone and in combination with MSC in nude mice bearing advanced human A253 and FaDu squamous cell carcinoma xenografts (B). (A) CDDP and oxaliplatin (OXAL) were administered by a single i.v. injection and irinotecan by weekly for 4 weeks. Se-methylselenocysteine at $0.75 \mathrm{mg}$ per rat per day p.o. daily for 21 days, with the first dose having started 14 days before CDDP or oxaliplatin treatment. For irinotecan group (16 rats) , MSC was administered daily for 14 days before and during irinotecan treatment for a total of 35 days. All treatments were initiated 14 days after tumour transplantation when the tumours reached $\sim 2500-3000 \mathrm{mg}$. In the oxaliplatin-alone $\left(10 \mathrm{mg} \mathrm{kg}^{-1}\right)$ group, 12 rats were evaluated, and in all other treatment groups, 4 rats were used. (B) $\bigcirc$ Untreated control; - MSC $0.2 \mathrm{mg}$ per mouse per day $\times 14 ; \boldsymbol{\Delta}$ CTX $100 \mathrm{mg} \mathrm{kg}^{-1}$ or CDDP $8 \mathrm{mg} \mathrm{kg}^{-1}$, i.v. $\times 1 ; \boldsymbol{C}$ CTX $100 \mathrm{mg} \mathrm{kg}^{-1}$ or CDDP $8 \mathrm{mg} \mathrm{kg}^{-1}$ (i.v. $\times 1$ ) + MSC $(0.2 \mathrm{mg}$ per mouse per day $\times 14)$. The treatment of CTX and CDDP was initiated on day 0 ( 7 days after tumour transplantation when the tumours reached $\sim 200-220 \mathrm{mg}$ ) and MSC by p.o. 7 days before and 7 days after CTX or CDDP in a total of 14 days. The mice were humanely killed when tumours reached $\sim 2000 \mathrm{mg}$. The numbers in parenthesis indicate the number of rats that achieved CR over the total number of rats treated.

The use of selenium as a modulator of chemotherapeutic agents to enhance the antitumour efficacy while simultaneously decreasing toxicities is a novel approach of recent origin (Cao et al, 2004). Previous reports from this laboratory have clearly demonstrated that MSC can synergistically modulate the therapeutic selectivity and efficacy of chemotherapeutic agents with different mechanisms of action in variety of xenograft models when used before and during treatment (Cao et al, 2004). Concurrent therapy without pretreatment with MSC had little effect on the treatment of anticancer drugs. The current study suggests that the protective effects of MSC from chemotherapeutic agent-induced damage on normal tissues provide the rationale for administering higher than

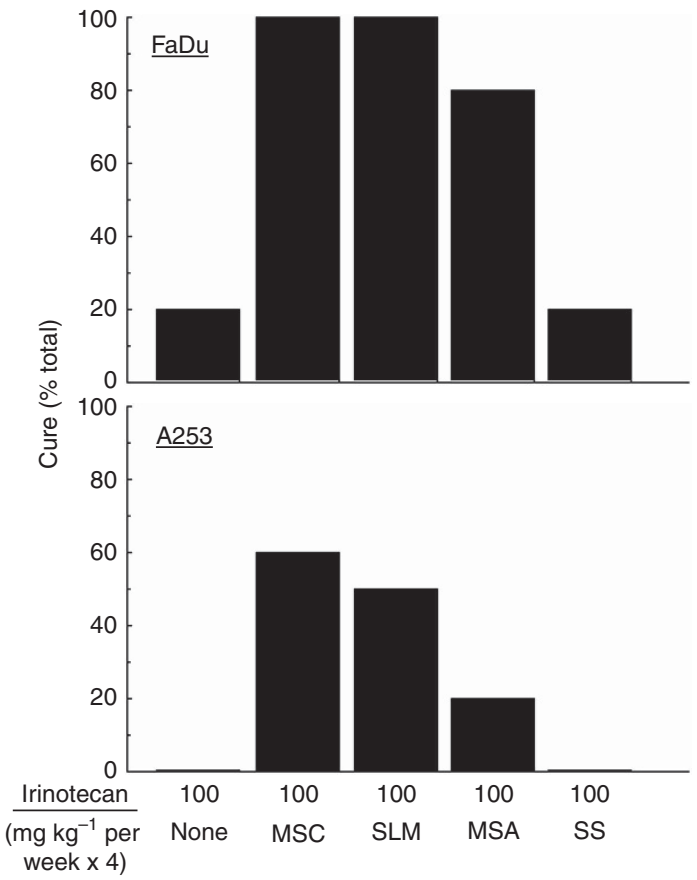

Figure 6. Antitumour activity of different types of selenium in combination with irinotecan in nude mice bearing advanced FaDu and A253 human head and neck tumour xenografts. All types of selenium (MSC, SLM, MSA, and SS) were administered orally at their respective maximum tolerated dose (MTD) daily for 7 days before and concurrent with the weekly administration of irinotecan for a total of 28 days. Five mice were used for each experimental group.

the MTD doses of anticancer drugs for potentially overcoming de novo drug resistance.

To evaluate and document the protective effect of MSC on clinical dose-limiting toxicities, four drugs of CTX, CDDP, oxaliplatin, and irinotecan were utilised. Our data clearly demonstrated the broad spectrum of protection by MSC of organ-specific toxicity induced by different types of chemotherapy. Bladder tissues were examined at 4 and $24 \mathrm{~h}$ after CTX treatment for bladder toxicity evaluation based on earlier report of cystitis appearing as early as $2 \mathrm{~h}$ post CTX in rodents (Anton, 2002). Results reported herein demonstrated that MSC is highly effective in protection from CTX-induced bladder toxicity (Figure 1).

Alopecia represents another major side effect of patients treated with CTX. Alopecia is caused by ablation of the rapidly dividing epithelium of the hair follicle. Currently, there is a lack of good animal model to study chemotherapy-induced alopecia. As it is difficult to demonstrate the direct protection effect of MSC on CTX-induced alopecia, we chose the method of hair regrowth after shaving to evaluate the effect of MSC. The data reported in Figure 3 clearly demonstrate the ability of MSC to allow normal hair growth in rats after CTX treatment.

The CDDP has been extensively used in the clinic for treatment of a variety of malignancies. It commonly causes renal, gastrointestinal, neuro, and haematological toxicities, with nephrotoxicity as the dose-limiting toxicity. The data in Figure 2 and Table 1 demonstrate the ability of MSC in protecting from higher dose of CDDP-induced body weight loss, diarrhoea, stomatitis, and lethality. Se-methylselenocysteine also markedly protected the kidneys from CDDP-induced damage. Treatment with CDDP at the MTD dramatically increased BUN and creatinine levels, whereas MSC restored BUN and creatinine to the normal levels (Table 1). Histologically, the kidney damage induced by CDDP was not observed in rats treated with MSC in combination with CDDP 
(Figure 2). Thus, MSC protects the function and histological feature of the kidney from CDDP-induced damage. There have been several reports of the ability of selenium to protect against CDDP-induced nephrotoxicity (Baldew et al, 1989; Satoh et al, 1989; Hu et al, 1997; Antunes et al, 2000, 2001; Camargo et al, 2001). However, sodium selenite only partially protected rats from CDDP-induced proximal tubular injury, and did not prevent deterioration of renal function (Camargo et al, 2001). This may be because sodium selenite, like other inorganic forms of selenium, was inferior to organoselenium compound such as the one used in our studies. Studies on the effect of more than 10 different forms of selenium carried out in our laboratory indicated that inorganic forms of selenium such as sodium selenite is less effective as a modulator of the antitumour activity of anticancer drugs against various human tumour xenografts (Figure 6 and YM Rustum, unpublished data). Activation of organoselenium compounds by $\beta$-lyase-type enzymes may play a role (Rooseboom et al, 2002). Unless MSC is activated, it is relatively nontoxic in vitro for up to $200 \mu \mathrm{M}$, a concentration $\sim 10$-fold higher than those achieved in vivo with $0.75 \mathrm{mg}$ per rat (YM Rustum, unpublished results).

Our previous study in nude mice bearing human tumour xenografts demonstrated that a minimum 7 days of pretreatment with MSC before chemotherapy was required for optimal enhancement of antitumour efficacy of irinotecan and protection from the drug-induced toxicity (Cao et al, 2004). In our current study, pretreatment with MSC before CTX, CDDP, oxaliplatin, and irinotecan treatment was necessary for optimal protection against drug-induced toxicities and for augmentation of the antitumour activity of these anticancer drugs. Concurrent treatment with MSC and CTX or CDDP did not offer significant protection against drug-induced toxicities and did not result in significant increase in antitumour activity of the agents under investigation (data not shown). Thus, daily treatment for a minimum of 7 days in the animals was confirmed critical for optimal selective protective effects and increasing antitumour efficacy. As discussed below, the results generated in this laboratory demonstrated that inhibition of HIF, a marker for angiogenesis and drug resistance, resulted in selective enhancement of drug delivery to tumour cells (Chintala et al, 2010, 2012; Bhattacharya et al, 2008, 2009), and these effects are selenium types, dose, and schedule dependent. Administration of chemotherapy at the time when optimal modulation effects by MSC was achieved is critical for therapeutic synergy with anticancer drugs evaluated in xenograft models.

The results reported herein extend and confirm the previous studies from this laboratory (Cao et al, 2004) demonstrating that MSC can significantly potentiate the antitumour efficacy of CTX, CDDP, oxaliplatin, and irinotecan against human FaDu and A253 HNSCCs (Figure 5B). Furthermore, we also demonstrated that MSC is effective in enhancing the antitumour activity of CDDP and oxaliplatin against advanced Ward colorectal carcinoma ( 2500-3000 mg, Figure 5A). The results reported herein support the original hypothesis that MSC will provide significant protection of various normal tissues from multiple drugs inducing a variety of organ-specific toxicities and will simultaneously enhance the antitumour activity of anticancer drugs.

The use of methionine $\alpha$-lyase (MET) has been evaluated and has demonstrated therapeutic potential in cancer therapy (Miki et al, 2001). The data generated in our laboratory confirmed these previous findings that selenomethionine and Se-methyl selenocysteine as prodrugs were relatively inactive in vitro and the cytotoxicities were significantly increased against a variety of human tumour cell lines after activation by methionase in vitro. However, in in vivo models, endogenous concentration of $\beta$-lyase is sufficient for activation of these selenium molecules to its presumed active moiety, methyl selenol. It is possible, however, that treatment with MET genes could further enhance the antitumour activity of selenium prodrugs, especially in the host and/or tumour with limited level of MET.

Recent report by the SELECT trial demonstrated that oral SLM (200 $\mu \mathrm{g}$ per day) did not prevent prostate cancer (Lippman et al, 2009). The lack of preventive effect of SLM on prostate cancer in this trial may be related to several factors and it should not deter further investigation of selenium. First, the dose of SLM used in this trial may be too low. We reported that the desired plasma concentration of selenium for optimal modulation of the therapeutic efficacy and selectivity of anticancer drugs in preclinical models is 20-30 $\mu \mathrm{M}$ (Fakih et al, 2006; Azrak et al, 2007), and this can be achieved by oral administration of SLM, twice daily, for 7 days at $7200 \mu \mathrm{g}$ per patient without toxicity (Fakih et al, 2008).

Although the mechanisms of action of MSC protection of normal organs/tissues and augmentation of the antitumour activity of anticancer drugs were not the subject of the present investigation, recent data suggest that MSC acts as an antioxidant against tumour tissues by targeting hypoxia-induced factor $1 \alpha$ (HIF-1 $\alpha$ ) through its main regulator prolyl hydroxylases (PHDs, Chintala et al, 2010, 2012). Furthermore, selenium is known to activate the expression of P53 and synchronisation of cells in the G phase (Botchkarev et al, 2000). The mechanism of MSC protection of normal tissue from drug-induced toxicities has not yet been documented.

For a drug to be effective and selective in cancer therapy, it must reach the target with sufficient concentrations to inhibit targets and related pathways that are responsible for tumour growth and/or metastasis. Second, the patients are relatively healthy men in this trial (Lippman et al, 2009) with no detectable tumours under normoxic condition, whereas our studies used advanced, hypoxic tumours (Chintala et al, 2010). Selenium was more cytotoxic under hypoxic condition than under normoxic condition. Enhanced cytotoxicity was associated with inhibition of HIF- $1 \alpha$ that is overexpressed in the majority of advanced solid tumours, including head and neck, colorectal, and renal cancers (Chintala et al, 2012). Third, our previous studies suggested that one of the possible mechanisms of selenium exhibiting its selective effects was associated with tumour angiogenesis and microenvironment (Bhattacharya et al, 2008, 2009). Selenium, therefore, like other antiangiogenic agents such as bevacizumab (Avastin) and cetuximab (Erbitux) used in the clinic demonstrate therapeutic synergy in combination with chemotherapeutic agents. Fourthly, not all forms of selenium are equally effective. We found that MSC is superior to SLM, sodium selenite, selenised yeast, and many other forms of selenium as selective modulator of the antitumour activity of anticancer drugs against various human tumour xenografts (Figure 6 and YM Rustum, unpublished data).

In brief, the results reported herein clearly demonstrated that MSC is a highly effective and selective modulator of the therapeutic efficacy and toxicity of various chemotherapeutic agents in animal models. This investigation is an extension of previous studies of selenium as a modulator of the efficacy and toxicity of anticancer drugs. Unlike other selenium-containing molecules, MSC requires one step activation by $\beta$-lyase with relatively less serum protein binding/interaction and the therapeutic concentrations can be achieved in vivo without toxicity. Se-methylselenocysteine is also much better than other antiangiogenic agents in clinical trial, is highly orally bioavailable, a potent inhibitor of HIF- $1 \alpha$ and HIF- $2 \alpha$ and their transcriptionally regulated VEGF and Glut-1, prevents the development of in vivo resistance to chemotherapy in preclinical models, and does not induce hypoxia (hypoxic cells are more sensitive to MSC than normoxic cells). Se-methylselenocysteine is relatively cheap and is orally bioavailable. It is important that the mechanisms of action of MSC alone and in combination with 
chemotherapy should be further investigated in order to develop the scientific rationale for clinical validation of hypothesis. There are critical end points in the design of future clinical trials to determine the optimal forms of selenium types and administration dose, schedule, and sequences in combination with chemotherapeutic agents that have direct cytotoxic effects against tumour.

\section{ACKNOWLEDGEMENTS}

This study was supported by Comprehensive Cancer Center Support Grant CA16056 from the National Cancer Institute, Bethesda, MD. We thank Dr Peter M Kanter for measurement of blood urea nitrogen and serum creatinine and Dr Arup B Bhattacharya for critical review of the manuscript.

\section{CONFLICT OF INTEREST}

The authors declare no conflict of interest.

\section{REFERENCES}

Alberts SR, Horvath WL, Sternfeld WC, Goldberg RM, Mahoney MR, Dakhil SR, Levitt R, Rowland K, Nair S, Sargent DJ, Donohue JH (2005) Oxaliplatin, fluorouracil, and leucovorin for patients with unresectable liver-only metastases from colorectal cancer: a North Central Cancer Treatment Group phase II study. J Clin Oncol 23: 9243-9249.

Amaral AF, Cantor KP, Silverman DT, Malats N (2010) Selenium and bladder cancer risk: a meta analysis. Cancer Epidemiol Biomarkers Prev 199: 2407-2415.

Anton E (2002) Delayed toxicity of cyclophosphamide on the bladder of DBA/2 and C57BL/6 female mouse. Int J Exp Pathol 83: 47-53.

Antunes LMG, Darin JD, Bianchi MDLP (2001) Effects of the antioxidants curcumin or selenium on cisplatin-induced nephrotoxicity and lipid peroxidation in rats. Pharmacol Res 43: 145-150.

Antunes LM, Francescato HD, Darin JD, de Lourdes P Bianchi M (2000) Effects of selenium pretreatment on cisplatin-induced chromosome aberrations in wister rats. Teratog Carcinog Mutagen 20: 341-348.

Azrak RG, Cao S, Pendyala L, Durrani FA, Prey J, Combs J, Fakih M, Rustum YM (2007) Plasma, normal and tumor tissue selenium concentrations as a function of 5-methyl-selenocysteine dose in mice bearing human tumor xenografts. Biochem Pharmacol 73: 1280-1287.

Baldew GS, Van Der Hamer CJ, Los G, Vermeulen NP, de Goeij JJ, McVie JG (1989) Selenium-induced protection against cis-diamminedichloroplatinum(II) nephrotoxicity in mice and rats. Cancer Res 49: 3020-3030.

Bhattacharya A, Seshadri M, Oven S, Toth K, Vaughan M, Rustum YM (2008) Tumor vascular maturation and improved drug delivery induced by Methyl-selenocysteine leads to therapeutic synergy with anticancer drugs. Clin Cancer Res 14: 3926-3932.

Bhattacharya A, Toth K, Sen A, Seshadri M, Cao S, Durrani F, Faber E, Repasky EA, Rustum YM (2009) Inhibition of colon cancer growth by MethylSelenocysteine-induced angiogenic chemomodulation is influenced by histologic characteristics of the tumor. Clin Colorectal Cancer 8: 155-162.

Bleiberg H (1998) Oxaliplatin (L-OHP): a new reality in colorectal cancer. Br J Cancer 77(Suppl 4): 1-3.

Botchkarev VA, Komarova EA, Siebenhaar F, Botchkareva NV, Komarov PG, Maurer M, Gilchrest BA, Gudkov AV (2000) p53 is essential for chemotherapy-induced hair loss. Cancer Res 60: 5002-5006.

Brinkman M, Reulen RC, Kellen E, Buntinx F, Zeegers MP (2006) Are men with low selenium levels at increased risk of prostate cancer? Eur J Cancer 42: 2463-2471.

Camargo SM, Francescato HD, Lavrador MA, Bianchi ML (2001) Oral administration of sodium selenite minimizes cisplatin toxicity on proximal tubules of rats. Biol Trace Elem Res 83: 251-262.

Cao S, Bhattacharya A, Durrani FA, Fakih M (2006) Irinotecan, oxaliplatin and raltitrexed for the treatment of advanced colorectal cancer. Expert Opin Pharmacother 7: 687-703.
Cao S, Durrani FA, Rustum YM (2004) Selective modulation of the therapeutic efficacy of anticancer drugs by selenium containing compounds against human tumor xenografts. Clin Cancer Res 10: 2561-2569.

Cao S, Rustum YM (2000) Synergistic antitumor activity of irinotecan in combination with 5-fluorouracil in rats bearing advanced colorectal cancer: role of drug sequence and dose. Cancer Res 60: 3717-3721.

Cao S, McGuire JJ, Rustum YM (1999) Antitumor activity of ZD1694 (Tomudex) against human head and neck cancer in dude mouse models: role of doing schedule and plasma thymidine. Clin Cancer Res 5: 1925-1934.

Chen CS, Lin J, Gross K, He YA, Halpert J, Waxman D (2004) Activation of the anticancer prodrugs cyclophosphamide and ifosfamide: identification of cytochrome P450 2B enzymes and site-specific mutants with improved enzyme kinetics. Mol Pharmacol 65: 1278-1287.

Chintala S, Toth K, Cao S, Durrani FA, Vaughan MM, Jenesen RL, Rustum YM (2010) Se-Methylselenocysteine sensitizes hypoxic tumor cells to irinotecan by targeting hypoxia- inducible factor $1 \alpha$. Cancer Chemother Pharmacol 66(5): 899-911.

Chintala S, Najrana T, Toth K, Cao S, Durrani FA, Pili R, Rustum YM (2012) Prolyl Hydroxylase 2-dependent and Von-Hippel-Lindu- independent degradation of hypoxia- inducible factor 1 and 2 alpha by selenium in clear cell renal cell carcinoma leads to tumor growth inhibition. BMC Cancer 12: 293.

Clark LC, Cantor KP, Allaway WH (1991) Selenium in forage crops and cancer mortality in U.S. counties. Arch Environ Health 46: 37-42.

Clark LC, Combs Jr GF, Turnbull BW, Slate EH, Chalker DK, Chow J, Davis LS, Glover RA, Graham GF, Gross EG, Krongrad A, Lesher Jr JL, Park HK, Sanders Jr BB, Smith CL, Taylor JR (1996) Effects of selenium supplementation for cancer prevention in patients with carcinoma of the skin: a randomized controlled trial. JAMA 276: 1957-1963.

Fakih MG, Pendyala L, Smith PF, Creaven PJ, Reid ME, Badmaev V, Azrak RG, Prey JD, Lawrence D, Rustum YM (2006) A phase I and pharmacokinetic study of fixed-dose selenomethionine and irintoecan in solid tumors. Clin Cancer Res 12: 1237-1244.

Fakih MG, Pendyala L, Brady W, Smith PF, Ross ME, Creaven PJ, Badmaev V, Prey JD, Rustum YM (2008) A phase I and pharmacokinetic study of selenomethionine in combination with a fixed dose of irinotecan in solid tumors. Cancer Chemother Pharmacol 62(3): 499-508.

Fraiser LH, Kanekal S, Kehrer JP (1991) Cyclophosphamide toxicity. Characterizing and avoiding the problem. Drugs 42: 781-795.

Hu YJ, Chen Y, Zhang YQ, Zhou MZ, Song XM, Zhang BZ, Luo L, Xu PM, Zhao YN, Zhao YB, Cheng G (1997) The protective role of selenium on the toxicity of cisplatin-contained chemotherapy regimen in cancer patients. Biol Trace Elem Res 56(3): 331-341.

Ibrahim A, Hirschfeld S, Cohen MH, Griebel DJ, Williams GA, Pazdur R (2004) FDA drug approval summaries: oxaliplatin. Oncologist 9: 8-12.

Ip C, Shu Z, Thompson HJ, Lisk D, Ganther HE (1999) Chemoprevention of mammary cancer with Se-allylselenocysteine and other selenoamino acids in the rats. Anticancer Res 19: 2875-2880.

Lippman SM, Klein EA, Goodman PJ, Lucia MS, Thompson IM, Ford LG, Parnes HL, Minasian LM, Gaziano JM, Hartline JA, Parsons JK, Bearden JD, Crawford ED, Goodman GE, Claudio J, Winquist E, Cook ED, Karp DD, Walther P, Lieber MM, Kristal AR, Darke AK, Arnold KB, Ganz PA, Santella RM, Albanes D, Taylor PR, Probstfield JL, Jagpal TJ, Crowley JJ, Meyskens Jr FL, Baker LH, Coltman CA Jr (2009) Effect of selenium and vitamin $\mathrm{E}$ on risk of prostate cancer and other cancers. The Selenium and Vitamin E Cancer Prevention Trial (SELECT). JAMA 301: 39-51.

Miki K, Xu M, Gupta A, Ba Y, Tan Y, Al-Refaie W, Bouvet M, Makuuchi M, Moossa AR, Hoffman RM (2001) Methioninase cancer gene therapy with selenomethionine as suicide prodrug substrate. Cancer Res 61: 6805-6810.

Miller LJ, Chandler SW, Ippoliti CM (1994) Treatment of cyclophosphamideinduced hemorrhagic cystitis with prostaglandins. Ann Pharmacother 28 : 590-594.

Rooseboom M, Schaaf G, Commandeur JN, Vermeulen NP, Fink-Gremmels J (2002) Beta- lyase-dependent attenuation of cisplatin-mediated toxicity by selenocysteine Se-conjugates in renal tubular cell lines. J Pharmacol Exp Ther 301: 884-892.

Satoh M, Naganuma A, Imura N (1989) Optimum schedule of selenium administration to reduce lethal and renal toxicities of cisdiamminedichloroplatinum in mice. J Pharmacobiodyn 12: 246-253.

Shepherd JD, Pringle LE, Barnett MJ, Klingemann HG, Reece DE, Phillips GL (1991) Mesna versus hyper hydration for the prevention of cyclophosphamide-induced hemorrhagic cystitis in bone marrow transplantation. J Clin Oncol 9: 2016-2020. 
Spayd RW, Bruschi B, Burdcik BA, Burdick BA, Dappen GM, Eikenberry JN, Esders TW, Figueras J, Goodhue CT, LaRossa DD, Nelson RW, Rand RN, Wu TW (1978) Multilayer film elements for clinical analysis: applications to representative chemical determinations. Clin Chem 24: 1343-1350.

Wasserman E, Sutherland W, Cvitkovic E (2001) Irinotecan plus oxaliplatin: a promising combination for advanced colorectal cancer. Clin Colorectal Cancer 1: 149-153.

Wilson RH, Lehky T, Thomas RR, Quinn MG, Floeter MK, Grem JL (2002) Acute oxaliplatin induced peripheral nerve hyperexcitability. J Clin Oncol 20: $1767-1774$.
Zhou H, Smith AH, Steinmaus C (2004) Selenium and lung cancer: a quantitative analysis of heterogeneity in the current epidemiological literature. Cancer Epidemiol Biomarkers Prev 13: 771-778.

This work is published under the standard license to publish agreement. After 12 months the work will become freely available and the license terms will switch to a Creative Commons AttributionNonCommercial-Share Alike 3.0 Unported License. 